\title{
The flag upper bound theorem for 3- and 5-manifolds
}

\author{
Hailun Zheng $\|^{t}$ \\ ${ }^{1}$ Department of Mathematics, University of Washington, Seattle, USA
}

\begin{abstract}
We prove that among all flag 3-manifolds on $n$ vertices, the join of two circles with $\left\lceil\frac{n}{2}\right\rceil$ and $\left\lfloor\frac{n}{2}\right\rfloor$ vertices respectively is the unique maximizer of the face numbers. This solves the first case of a conjecture due to Lutz and Nevo. Further, we establish a sharp upper bound on the number of edges of flag 5-manifolds and characterize the cases of equality. We also show that the inequality part of the flag upper bound conjecture continues to hold for all flag 3-dimensional Eulerian complexes and characterize the cases of equality in this class.

Résumé. Nous montrons que parmi tous les 3-variétés à $n$ sommets, le join de deux cercles avec $\left\lceil\frac{n}{2}\right\rceil$ et $\left\lfloor\frac{n}{2}\right\rfloor$ sommets respectivement est la seule variété qui maximise le nombre de faces. Cela prouve le premier cas d'une conjecture due à Lutz et Nevo. Par ailleurs, nous établissons une borne supérieure optimale pour le nombre d'arêtes des 5 -variétés de cliques et nous caractérisons les cas d'égalité. Nous montrons également que l'inégalité de la conjecture de la borne supérieure est satisfaite pour toutes les complexes de cliques Eulériens 3-dimensionnels et nous caractérisons les cas d'égalité dans cette classe.
\end{abstract}

Keywords. Upper bound theorem, Flag complex, Eulerian complex, Simplicial manifold

\section{Introduction}

One of the classical problems in geometric combinatorics deals with the following question: for a given class of simplicial complexes, find tight upper bounds on the number of $i$-dimensional faces as a function of the number of vertices and the dimension. Since Motzkin (1957) proposed the upper bound conjecture (UBC, for short) for polytopes in 1957, this problem has been solved for various families of complexes. In particular, McMullen (1970) and Stanley (1975) proved that neighborly polytopes simultaneously maximize all the face numbers in the class of polytopes and simplicial spheres. However, it turns out that, apart from cyclic polytopes, many other classes of neighborly spheres or even neighborly polytopes exist, see Shermer (1982) and Padrol (2013) for examples and constructions of neighborly polytopes.

A simplicial complex $\Delta$ is flag if all of its minimal non-faces have cardinality two, or equivalently, $\Delta$ is the clique complex of its graph. Flag complexes form a beautiful and important class of simplicial complexes. For example, barycentric subdivisions of simplicial complexes, order complexes of posets, and Coxeter complexes are flag complexes. Despite a lot of effort that went into studying the face numbers

\footnotetext{
$\dagger$ Email: hailunz@uw.edu. The author was partially supported by a graduate fellowship from NSF grant DMS-1361423. 
of flag spheres, in particular in relation with the Charney-Davis conjecture (Charney and Davis 1995), and its generalization given by Gal's conjecture (Gal, 2005), a flag upper bound theorem for spheres is still unknown. The upper bounds of face numbers for general simplicial $(d-1)$-spheres are far from sharp for those of flag $(d-1)$-spheres, since the graph of any flag $(d-1)$-dimensional complex is $K_{d+1}$-free. Gal (2005) established the upper bound theorem for flag spheres of dimension less than five. However, starting from dimension five, there are only conjectural upper bounds. For $m \geq 1$, we let $J_{m}(n)$ be the $(2 m-1)$-sphere on $n$ vertices obtained as the join of $m$ copies of the circle, each one a cycle with either $\left\lfloor\frac{n}{m}\right\rfloor$ or $\left\lceil\frac{n}{m}\right\rceil$ vertices. We also let $J_{m}^{*}(n)$ be the $2 m$-sphere on $n$ vertices defined as the suspension of $J_{m}(n-2)$.

Conjecture 1.1 (Nevo and Petersen 2010 Conjecture 6.3) If $\Delta$ is a flag homology sphere, then $\gamma(\Delta)$ satisfies the Frankl-Füredi-Kalai inequalities on $\left\lfloor\frac{\operatorname{dim} \Delta+1}{2}\right\rfloor$-colored complexes. In particular, if $\Delta$ is of dimension $2 m-1$, where $m \geq 2$, then $f_{i}(\Delta) \leq f_{i}\left(J_{m}(n)\right)$ for $1 \leq i \leq 2 m-1$; if $\Delta$ is of dimension $2 m$, where $m \geq 1$, then $f_{i}(\Delta) \leq f_{i}\left(J_{m}^{*}(n)\right)$ for $1 \leq i \leq 2 m$.

As for the case of equality, Lutz and Nevo posited that as opposed to the case of all simplicial spheres, for a fixed dimension $2 m-1$ and the number of vertices $n$, there is only one maximizer of the face numbers.

Conjecture 1.2 Lutz and Nevo (2014 Conjecture 6.3) Let $m \geq 2$ and $\Delta$ be a flag simplicial $(2 m-1)$ sphere on $n$ vertices. Then $f_{i}(\Delta)=f_{i}\left(J_{m}(n)\right)$ for some $1 \leq i \leq m$ if and only if $\Delta=J_{m}(n)$.

Recently, Adamaszek and Hladký (2015) proved that this conjecture holds asymptotically for flag homology manifolds. Several celebrated theorems from extremal graph theory served as tools for their work. As a result, the proof simultaneously gives upper bounds on $f$-numbers, $h$-numbers, $g$-numbers and $\gamma$ numbers, but it only applies to flag homology manifolds with an extremely large number of vertices.

Our first main result is that both Conjecture 1.1 and Conjecture 1.2 hold for all flag 3-manifolds.

Theorem 1.3 Let $\Delta$ be a flag 3-manifold on $n$ vertices. Then $f_{i}(\Delta) \leq f_{i}\left(J_{2}(n)\right)$. If equality holds for some $1 \leq i \leq 3$, then $\Delta=J_{2}(n)$.

The proof of Theorem 1.3 only relies on simple properties of flag complexes and Eulerian complexes. We also establish an analogous result on the number of edges of flag 5-manifolds.

Theorem 1.4 Let $\Delta$ be a flag homology 5-manifold on $n$ vertices. Then $f_{1}(\Delta) \leq f_{1}\left(J_{3}(n)\right)$. Equality holds if and only if $\Delta=J_{3}(n)$.

In 1964, Klee (1964) proved that Motzkin's UBC for polytopes holds for a much larger class of Eulerian complexes as long as they have sufficiently many vertices, and conjectured that the UBC holds for all Eulerian complexes. Our second main result deals with flag Eulerian complexes, and asserts that Conjecture 1.1 continues to hold for all 3-dimensional flag Eulerian complexes.

Theorem 1.5 Let $\Delta$ be a 3-dimensional flag Eulerian complex on $n$ vertices. Then $f_{i}(\Delta) \leq f_{i}\left(J_{2}(n)\right)$ for $1 \leq i \leq 3$.

This provides supporting evidence to a question of Adamaszek and Hladký (2015. Problem 17(i)) in the case of dimension 3, where they proposed that Conjecture 1.1 holds for all odd-dimensional flag weak pseudomanifolds with sufficiently many vertices. We also give constructions of the maximizers of face numbers in this class and show that they are the only maximizers. Our proof is based on an application of the inclusion-exclusion principle and double counting. 
The Extended Abstract is organized as follows. In Section 2, we discuss basic facts on simplicial complexes and flag complexes. In Section 3, we provide the proof of our first main result asserting that given a number of vertices $n$, the maximum face numbers of a flag 3-manifold are achieved only when this manifold is the join of two circles of length as close as possible to $\frac{n}{2}$. In Section 4, we apply an analogous argument to the class of flag 5-manifolds. In Section 5, we show that the same upper bounds continue to hold for the class of 3-dimensional flag Eulerian complexes, and discuss the maximizers of the face numbers in this class. Finally, we close in Section 6 with some concluding remarks.

\section{Preliminaries}

A simplicial complex $\Delta$ on a vertex set $V=V(\Delta)$ is a collection of subsets $\sigma \subseteq V$, called faces, that is closed under inclusion. For $\sigma \in \Delta$, let $\operatorname{dim} \sigma:=|\sigma|-1$ and define the dimension of $\Delta, \operatorname{dim} \Delta$, as the maximal dimension of its faces. A facet in $\Delta$ is a maximal face under inclusion, and we say that $\Delta$ is pure if all of its facets have the same dimension.

If $\Delta$ is a simplicial complex and $\sigma$ is a face of $\Delta$, the link of $\sigma$ in $\Delta$ is $\mathrm{lk}_{\Delta} \sigma:=\{\tau-\sigma \in \Delta: \sigma \subseteq \tau \in$ $\Delta\}$, and the deletion of a vertex set $W$ from $\Delta$ is $\Delta \backslash W:=\{\sigma \in \Delta: \sigma \cap W=\emptyset\}$. The restriction of $\Delta$ to a vertex set $W$ is defined as $\Delta[W]:=\{\sigma \in \Delta: \sigma \subseteq W\}$. If $\Delta$ and $\Gamma$ are two simplicial complexes on disjoint vertex sets, then the join of $\Delta$ and $\Gamma$, denoted as $\Delta * \Gamma$, is the simplicial complex on vertex set $V(\Delta) \cup V(\Gamma)$ whose faces are $\{\sigma \cup \tau: \sigma \in \Delta, \tau \in \Gamma\}$.

A simplicial complex $\Delta$ is a simplicial manifold (resp. simplicial sphere) if the geometric realization of $\Delta$ is homeomorphic to a manifold (resp. sphere). We denote by $\tilde{H}_{*}(\Delta ; \mathbf{k})$ the reduced homology of $\Delta$ computed with coefficients in a field $\mathbf{k}$, and by $\beta_{i}(\Delta ; \mathbf{k}):=\operatorname{dim}_{\mathbf{k}} \tilde{H}_{i}(\Delta ; \mathbf{k})$ the reduced Betti numbers of $\Delta$ with coefficients in $\mathbf{k}$. We say that $\Delta$ is a $(d-1)$-dimensional $\mathbf{k}$-homology manifold if $\tilde{H}_{*}\left(\mathrm{lk}_{\Delta} \sigma ; \mathbf{k}\right) \cong \tilde{H}_{*}\left(\mathbb{S}^{d-1-|\sigma|} ; \mathbf{k}\right)$ for every nonempty face $\sigma \in \Delta$. A $\mathbf{k}$-homology sphere is a k-homology manifold that has the $\mathbf{k}$-homology of a sphere. Every simplicial manifold (resp. simplicial sphere) is a homology manifold (resp. homology sphere). Moreover, in dimension two, the class of homology 2spheres coincides with that of simplicial 2-spheres, and hence in dimension three, the class of homology 3-manifolds coincides with that of simplicial 3-manifolds.

For a $(d-1)$-dimensional complex $\Delta$, we let $\chi(\Delta):=\sum_{i=0}^{d-1}(-1)^{i} \beta_{i}(\Delta ; \mathbf{k})$ be the reduced Euler characteristic of $\Delta$. A simplicial complex $\Delta$ is called an Eulerian complex if $\Delta$ is pure and $\chi\left(\mathrm{lk}_{\Delta} \sigma\right)=$ $(-1)^{\operatorname{dim} l \mathrm{k}_{\Delta} \sigma}$ for every $\sigma \in \Delta$, including $\sigma=\emptyset$. In particular, it follows from the Poincaré duality theorem that all odd-dimensional simplicial manifolds are Eulerian.

A $(d-1)$-dimensional simplicial complex $\Delta$ is called a weak $(d-1)$-pseudomanifold if it is pure and every $(d-2)$-face (called ridge) of $\Delta$ is contained in exactly two facets. A weak $(d-1)$-pseudomanifold $\Delta$ is called a normal $(d-1)$-pseudomanifold if it is connected, and the link of each face of dimension $\leq d-3$ is also connected. Every Eulerian complex is a weak pseudomanifold, and every connected homology manifold is a normal pseudomanifold. In fact, every normal 2-pseudomanifold is also a homology 2manifold. However, for $d>3$, the class of normal $(d-1)$-pseudomanifolds is much larger than the class of homology $(d-1)$-manifolds. It is well-known that if $\Delta$ is a weak (resp. normal) $(d-1)$ pseudomanifold and $\sigma$ is a face of $\Delta$ of dimension at most $d-2$, then the link of $\sigma$ is also a weak (resp. normal) pseudomanifold. The following lemma gives another property of normal pseudomanifolds, see Bagchi and Datta (2008, Lemma 1.1).

Lemma 2.1 Let $\Delta$ be a normal $(d-1)$-pseudomanifold, and let $W$ be a subset of vertices of $\Delta$ such that 
the induced subcomplex $\Delta[W]$ is a normal $(d-2)$-pseudomanifold. Then the induced subcomplex of $\Delta$ on vertex set $V(\Delta) \backslash W$ has at most two connected components.

For a $(d-1)$-dimensional complex $\Delta$, we let $f_{i}=f_{i}(\Delta)$ be the number of $i$-dimensional faces of $\Delta$ for $-1 \leq i \leq d-1$. The vector $\left(f_{-1}, f_{0}, \cdots, f_{d-1}\right)$ is called the $f$-vector of $\Delta$. Since the graph of any simplicial 2-sphere is a maximal planar graph, it follows that the $f$-vector of a simplicial 2-sphere is uniquely determined by $f_{0}$. For a 3-dimensional Eulerian complex, the following lemma indicates that its $f$-vector is uniquely determined by $f_{0}$ and $f_{1}$. (We omit the proof.)

Lemma 2.2 The f-vector of a 3-dimensional Eulerian complex satisfies

$$
\left(f_{0}, f_{1}, f_{2}, f_{3}\right)=\left(f_{0}, f_{1}, 2 f_{1}-2 f_{0}, f_{1}-f_{0}\right)
$$

A simplicial complex $\Delta$ is flag if all minimal non-faces of $\Delta$, also called missing faces, have cardinality two; equivalently, $\Delta$ is the clique complex of its graph. The following lemma (Nevo and Petersen, 2010 , Lemma 5.2) gives a basic property of flag complexes:

Lemma 2.3 Let $\Delta$ be a flag complex on vertex set $V$. If $W \subseteq V$, then $\Delta[W]$ is also flag. Furthermore, if $\sigma$ is a face in $\Delta$, then $\mathrm{lk}_{\Delta} \sigma=\Delta\left[V\left(\mathrm{lk}_{\Delta} \sigma\right)\right]$. In particular, all links in a flag complex are also flag.

Finally, we recall some terminology from graph theory. A graph $G$ is a path graph if the set of its vertices can be ordered as $x_{1}, x_{2}, \cdots, x_{n}$ in such a way that $\left\{x_{i}, x_{i+1}\right\}$ is an edge for all $1 \leq i \leq n-1$ and there are no other edges. Similarly, a cycle graph is a graph obtained from a path graph by adding an edge between the end points of the path.

\section{The Proof of flag UBC for flag 3-manifolds}

Recall that in the introduction, we defined $J_{m}(n)$ to be the $(2 m-1)$-sphere on $n$ vertices obtained as the join of $m$ circles, each one of length either $\left\lfloor\frac{n}{m}\right\rfloor$ or $\left\lceil\frac{n}{m}\right\rceil$. The goal of this section is to prove the flag UBC for flag 3-manifolds (see Conjectures 1.1 and 1.2). We start with the following lemma.

Lemma 3.1 Let $\Delta$ be a flag normal 3-pseudomanifold on $n$ vertices. Then $f_{1}(\Delta) \leq f_{1}\left(J_{2}(n)\right)+c$, where $c=3-3 \min _{v \in \Delta} \chi\left(\mathrm{lk}_{\Delta} v\right)$.

Proof: Let $v$ be a vertex of maximum degree in $V(\Delta)$. We let $a=f_{0}\left(\mathrm{lk}_{\Delta} v\right), W_{1}=V\left(\mathrm{lk}_{\Delta} v\right)$ and $W_{2}=V(\Delta) \backslash V\left(\mathrm{lk}_{\Delta} v\right)$. Since $\Delta$ is a normal 3-pseudomanifold, $\mathrm{lk}_{\Delta} v$ is a normal 2-pseudomanifold, i.e., a simplicial 2-manifold. Furthermore, since $\Delta$ is flag, by Lemma 2.3 , $\mathrm{lk}_{\Delta} v$ is the restriction of $\Delta$ to $W_{1}$. Thus, by Lemma 2.1, the induced subcomplex $\Delta\left[W_{2}\right]$ has at most two connected components. Since $v$ is not connected to any vertices in $W_{2} \backslash\{v\}$, it follows that $\{v\}$ and $\Delta\left[W_{2} \backslash\{v\}\right]$ are the two connected components in $\Delta\left[W_{2}\right]$. 
We now count the edges of $\Delta$. They consist of the edges of $\Delta\left[W_{1}\right]=\mathrm{lk}_{\Delta} v$, the edges of $\Delta\left[W_{2}\right]$ and the edges between these two sets. In addition, $\sum_{w \in W_{2}} f_{0}\left(\mathrm{lk}_{\Delta} w\right)$ counts the edges of $\Delta\left[W_{2}\right]$ twice. Thus,

$$
\begin{aligned}
f_{1}(\Delta) & =f_{1}\left(\Delta\left[W_{1}\right]\right)+\left(\sum_{w \in W_{2}} f_{0}\left(\mathrm{l}_{\Delta} w\right)\right)-f_{1}\left(\Delta\left[W_{2}\right]\right) \\
& \stackrel{(*)}{\leq} f_{1}\left(\mathrm{lk}_{\Delta} v\right)+\left|W_{2}\right| \cdot \max _{w \in W_{2}} f_{0}\left(\mathrm{lk}_{\Delta} w\right)-\left(f_{0}\left(\Delta\left[W_{2} \backslash\{v\}\right]\right)-1\right) \\
& \stackrel{(* *)}{=}\left(3 a-6+3\left(1-\chi\left(\mathrm{lk}_{\Delta} v\right)\right)\right)+(n-a) a-(n-a-2) \\
& =-a^{2}+a(n+4)-(n+4)+3-3 \chi\left(\mathrm{lk}_{\Delta} v\right) \\
& \stackrel{(* * *)}{\leq}\left\lfloor\frac{n^{2}}{4}\right\rfloor+n+3-3 \chi\left(\mathrm{lk}_{\Delta} v\right) \\
& =f_{1}\left(J_{2}(\Delta)\right)+3\left(1-\chi\left(\mathrm{lk}_{\Delta} v\right)\right) .
\end{aligned}
$$

Here in (*) we used that $\Delta\left[W_{2} \backslash\{v\}\right]$ is connected and hence has at least $f_{0}\left(\Delta\left[W_{2} \backslash\{v\}\right]\right)-1$ edges. Equality (**) follows from the fact that $\mathrm{lk}_{\Delta} v$ is a 2 -manifold with $a$ vertices, and (***) is obtained by optimizing the function $p(a)=-a^{2}+a(n+4)$. Hence the result follows.

Proof of Theorem 1.3: We use the same notation as in the proof of Lemma 3.1 That is, we let $v$ be a vertex of maximum degree in $V(\Delta)$. We let $a=f_{0}\left(\mathrm{lk}_{\Delta} v\right), W_{1}=V\left(\mathrm{lk}_{\Delta} v\right)$ and $W_{2}=V(\Delta) \backslash V\left(\mathrm{lk}_{\Delta} v\right)$. Since $\Delta$ is a flag 3-manifold, $\chi\left(\operatorname{lk}_{\Delta} w\right)=1$ for every $w \in \Delta$. Hence by Lemma 3.1, $f_{1}(\Delta) \leq f_{1}\left(J_{2}(\Delta)\right)$. Furthermore, it follows from steps $(*)$ and $(* * *)$ in equality $\sqrt{1}$ that $f_{1}(\Delta)=f_{1}\left(J_{2}(n)\right)$ holds only if $f_{0}\left(\operatorname{lk}_{\Delta} w\right)=a=\left\lceil\frac{n+4}{2}\right\rceil$ or $\left\lfloor\frac{n+4}{2}\right\rfloor$ for all $w \in W_{2}$, and $\Delta\left[W_{2} \backslash\{w\}\right]$ is a tree.

We claim that if $f_{1}(\Delta)=f_{1}\left(J_{2}(n)\right)$, then $\Delta=J_{2}(n)$. This indeed holds if $n=8$ or 9 , since the only flag 3-manifolds on 8 or 9 vertices are $J_{2}(8)$ and $J_{2}(9)$. Next we assume that $n \geq 10$, where $\left|W_{2}\right|=n-a \geq\left\lceil\frac{n}{2}\right\rceil-2>2$. Hence the tree $\Delta\left[W_{2} \backslash\{v\}\right]$ has at least one edge, and thus there is a vertex $u_{1} \in W_{2}$ such that $\operatorname{deg}_{\Delta\left[W_{2}\right]} u_{1}=1$. Let $u_{2}$ be the unique vertex in $W_{2}$ that is connected to $u_{1}$. Since $f_{0}\left(\mathrm{lk}_{\Delta} u_{1}\right)=a$, the vertex $u_{1}$ must be connected to all vertices in $W_{1}$ except for one vertex. We let $z_{1}$ be this vertex and denote the circle $\operatorname{lk}_{\mathrm{lk}_{\Delta} v} z_{1}$ by $C_{1}$. Since $\Delta$ is flag, $\operatorname{lk}_{\Delta} u_{1} \supseteq \Delta\left[W_{1} \backslash\left\{z_{1}\right\}\right]=$ $\mathrm{lk}_{\Delta} v-\left\{z_{1}\right\} * C_{1}$, and hence

$$
\mathrm{lk}_{\Delta} u_{1}=\left(\mathrm{lk}_{\Delta} v-\left\{z_{1}\right\} * C_{1}\right) \cup\left(\left\{u_{2}\right\} * C_{1}\right) .
$$

If $\left\{z_{1}\right\} \in \mathrm{lk}_{\Delta} u_{2}$, then $\operatorname{lk}_{\Delta} u_{2} \supseteq C_{1} *\left\{u_{1}, z_{1}\right\}$. Since $C_{1} *\left\{u_{1}, z_{1}\right\}$ is a 2 -sphere, it follows that $\operatorname{lk}_{\Delta} u_{2}=C_{1} *\left\{u_{1}, z_{1}\right\}$ and $f_{0}\left(C_{1}\right)=a-2$. Hence $W_{2}=\left\{u_{1}, u_{2}\right\}$ and $W_{1}=V\left(C_{1}\right) \cup\left\{z_{1}\right\} \cup$ $\left\{z_{2}\right\}$ for some vertex $z_{2} \in W_{1}$, so that $\mathrm{lk}_{\Delta} v=\left\{z_{1}, z_{2}\right\} * C_{1}$. Now assume that $\left\{z_{1}\right\} \notin 1 \mathrm{k}_{\Delta} u_{2}$ and $u_{2}$ is connected to vertices $u_{3}, u_{4}, \cdots, u_{k}$ in $\Delta\left[W_{2}\right]$. Since $C_{1}$ is a circle in the 2-sphere $1 \mathrm{k}_{\Delta} u_{2}$, the subcomplex $\mathrm{lk}_{\Delta} u_{2} \backslash V\left(C_{1}\right)$ has two contractible connected components. If there is a vertex $u_{i}$ such that $\operatorname{lk}_{\mathrm{lk}_{\Delta} u_{2}} u_{i}=C_{1}$, then $\operatorname{lk}_{\Delta} u_{2} \supseteq C_{1} *\left\{u_{1}, u_{i}\right\}$ and hence this link is exactly $C_{1} *\left\{u_{1}, u_{i}\right\}$. This implies that $\operatorname{deg}_{\Delta\left[W_{2}\right]} u_{2}=2$. Otherwise, if $\operatorname{lk}_{\mathrm{lk}_{\Delta} u_{2}} u_{i} \neq C_{1}$ for all $3 \leq i \leq k$, then each $u_{i}$ is connected to at least one vertex in $\operatorname{lk}_{\Delta} v \backslash\left(V\left(C_{1}\right) \cup\left\{z_{1}\right\}\right)$. Since $\operatorname{lk}_{\Delta} u_{1} \supseteq \operatorname{lk}_{\Delta} v \backslash\left\{z_{1}\right\}$, it follows that the vertices $u_{1}$ and $u_{3}, \cdots, u_{k}$ are in the same connected component, and hence $l_{\mathrm{k}_{\Delta}} u_{2} \backslash V\left(C_{1}\right)$ is connected, a contradiction.

By applying the above argument inductively, we obtain that $\Delta\left[W_{2} \backslash\{v\}\right]$ is a path graph $u_{1}, u_{2}, \cdots, u_{n-a-1}$, and there is a vertex $z_{2}$ in $W_{1}$ such that $\operatorname{lk}_{\Delta} u_{1}=\left\{z_{2}, u_{2}\right\} * C_{1}$ and $\operatorname{lk}_{\Delta} v=C_{1} *\left\{z_{1}, z_{2}\right\}$. Furthermore, 
$C_{1} \subseteq \mathrm{lk}_{\Delta} u_{i}$ for all $u_{i} \in W_{2}$. Then we let $C_{2}$ be the cycle graph $\left(v, z_{2}, u_{1}, u_{2}, \cdots, u_{n-a-1}, z_{1}\right)$. It follows that $\Delta=C_{1} * C_{2}$. Since $a=\left|C_{1}\right|+2=\left\lfloor\frac{n+4}{2}\right\rfloor$ or $\left\lceil\frac{n+4}{4}\right\rceil, C_{1}$ and $C_{2}$ must be cycles of length $\left\lfloor\frac{n}{2}\right\rfloor$ or $\left\lceil\frac{n}{2}\right\rceil$. This implies $\Delta=J_{2}(n)$.

By Lemma 2.2, the value of $f_{2}$ or $f_{3}$ determines $f_{1}$, and if either of them is maximal, then also $f_{1}$ is maximal. This yields the result.

\section{Counting edges of flag homology 5-manifolds}

Recall that we use $J_{m}^{*}(n)$ to denote the suspension of $J_{m}(n-2)$. For even-dimensional flag homology spheres, the following is a special case of the last part of Conjecture 1.1

Conjecture 4.1 Fix $m \geq 1$. For every flag homology $2 m$-sphere $\Delta$ on $n$ vertices, we have $f_{1}(M) \leq$ $f_{1}\left(J_{m}^{*}(n)\right)$.

Using the techniques similar to those in Section 3, we establish the following proposition.

Proposition 4.2 Let $\Delta$ be a flag $(2 m-1)$-manifold on $n$ vertices. If Conjecture 4.1 holds for all flag homology $2 i$-spheres with $1 \leq i \leq m-1$, then $f_{1}(\Delta) \leq f_{1}\left(J_{m}(n)\right)$. Equality holds only when $\Delta=$ $J_{m}(n)$.

Proof: A careful adaptation of the argument in Lemma 3.1 and Theorem 1.3 yields the result.

Proof of Theorem 1.4: The result follows from the fact that Conjecture 4.1 is known to hold in the case of dimension four (see Gal, 2005, Theorem 3.1.3).

\section{The face numbers of 3-dimensional flag Eulerian complexes}

In Lemma 3.1. we established an upper bound on the number of edges for all flag normal 3-pseudomanifolds. In this section, we find tight upper bounds on the face numbers for all 3-dimensional flag Eulerian complexes. The proof relies on the following three lemmas.

Lemma 5.1 Let $\Delta$ be a flag $(d-1)$-dimensional simplicial complex.

(a) If $\sigma_{1}$ and $\sigma_{2}$ are two ridges that lie in the same facet $\sigma$ in $\Delta$, then the links of $\sigma_{1}$ and $\sigma_{2}$ are disjoint.

(b) If $\sigma=\tau_{1} \sqcup \tau_{2}$ is a face in $\Delta$, then $V\left(\mathrm{lk}_{\Delta} \tau_{1}\right) \cap V\left(\mathrm{lk}_{\Delta} \tau_{2}\right)=V\left(\mathrm{lk}_{\Delta} \sigma\right)$. In particular, if $\sigma$ is a facet, then $f_{0}\left(\mathrm{lk}_{\Delta} \tau_{1}\right)+f_{0}\left(\mathrm{lk}_{\Delta} \tau_{2}\right) \leq f_{0}(\Delta)$.

The proof follows from the definition of flag complexes. We omit it for the sake of brevity. Lemma 5.1 part (b) implies that if $\Delta$ is a flag 3-dimensional simplicial complex and $\sigma \in \Delta$ is a facet, then $\sum_{e \subseteq \sigma} f_{0}\left(\mathrm{lk}_{\Delta} e\right) \leq 3 f_{0}(\Delta)$, where the sum is over the edges of $\sigma$. The following lemma suggests a better estimate on $\sum_{e \subseteq \sigma} f_{0}\left(\mathrm{lk}_{\Delta} e\right)$ for an arbitrary flag weak 3-pseudomanifold $\Delta$.

Lemma 5.2 Let $\Delta$ be a flag weak 3-pseudomanifold on $n$ vertices. Then for any facet $\sigma=\left\{v_{1}, v_{2}, v_{3}, v_{4}\right\}$ in $\Delta, \sum_{e \subseteq \sigma} f_{0}\left(\mathrm{lk}_{\Delta} e\right) \leq n+16$, where the sum is over the edges of $\sigma$. If equality holds, then $\cup_{w \in \tau} V\left(\mathrm{k}_{\Delta} w\right)=$ $V(\Delta)$ for any ridge $\tau \subseteq \sigma$. 
Proof: Let $V_{i}=V\left(\mathrm{lk}_{\Delta} v_{i}\right)$ for $1 \leq i \leq 4$. By Lemma 5.1 part (b), for any distinct $1 \leq i, j \leq$ 4, we have $V_{i} \cap V_{j}=V\left(\operatorname{lk}_{\Delta}\left\{v_{i}, v_{j}\right\}\right)$ and $V_{1} \cap V_{2} \cap V_{3} \cap V_{4}=V\left(\operatorname{lk}_{\Delta} \sigma\right)=\emptyset$. Also since $\Delta$ is a weak 3-pseudomanifold, any ridge of $\Delta$ is contained in exactly two facets. Hence $V_{i} \cap V_{j} \cap V_{k}=$ $V\left(\operatorname{lk}_{\Delta}\left\{v_{i}, v_{j}, v_{k}\right\}\right)$ is a set of cardinality two. By the inclusiong-exclusion principle, we obtain that

$$
\begin{aligned}
\sum_{1 \leq i<j \leq 4}\left|V_{i} \cap V_{j}\right| & =-\left|V_{1} \cup V_{2} \cup V_{3} \cup V_{4}\right|+\sum_{1 \leq i \leq 4}\left|V_{i}\right|+\sum_{1 \leq i<j<k \leq 4}\left|V_{i} \cap V_{j} \cap V_{k}\right|-\left|V_{1} \cap V_{2} \cap V_{3} \cap V_{4}\right| \\
& =\sum_{1 \leq i \leq 4}\left|V_{i}\right|-\left|V_{1} \cup V_{2} \cup V_{3} \cup V_{4}\right|+\left(\begin{array}{c}
4 \\
3
\end{array}\right) \cdot 2 \\
& =\left(\left|V_{1}\right|+\left|V_{2}\right|-\left|V_{1} \cup V_{2}\right|\right)+\left(\left|V_{3}\right|+\left|V_{4}\right|-\left|V_{3} \cup V_{4}\right|\right)+\left|\left(V_{1} \cup V_{2}\right) \cap\left(V_{3} \cup V_{4}\right)\right|+8 \\
& =\left|V_{1} \cap V_{2}\right|+\left|V_{3} \cap V_{4}\right|+\left|\left(V_{1} \cup V_{2}\right) \cap\left(V_{3} \cup V_{4}\right)\right|+8 .
\end{aligned}
$$

For simplicity, we denote the set $\left(V_{1} \cup V_{2}\right) \cap\left(V_{3} \cup V_{4}\right)$ as $\bar{V}$. Notice that by Lemma 5.1 part (b), any vertex $v \in \Delta$ belongs to at most one of the sets $V_{1} \cap V_{2}$ and $V_{3} \cap V_{4}$. We split the vertices of $\Delta$ into the following three types.

1. If $v \in V_{1} \cap V_{2}$ and $v \notin V_{3} \cup V_{4}$, or if $v \in V_{3} \cap V_{4}$ and $v \notin V_{1} \cup V_{2}$, then $v \notin \bar{V}$. Each of these vertices contributes 1 to the right-hand side of 2 .

2. If $v \in V_{i} \cap V_{j} \cap V_{k}$ for some triple $\{i, j, k\} \subseteq[4]$, then $v$ belongs to either $V_{1} \cap V_{2}$ or $V_{3} \cap V_{4}$, and $v \in \bar{V}$. By Lemma 5.1 part (a), every pair of ridges in $\sigma$ has disjoint links. Since $\left|V_{i} \cap V_{j} \cap V_{k}\right|=2$, the number of such vertices is exactly 8 , and each of them contributes 2 to the right-hand side of (2).

3. If $v \notin V_{1} \cap V_{2}$ and $v \notin V_{3} \cap V_{4}$, then $v$ contributes to the right-hand side of (2) at most 1 . This case occurs only when $v \in \bar{V}$, that is, when $v$ belongs to one of $V_{1}$ and $V_{2}$, and one of $V_{3}$ and $V_{4}$.

Hence $\sum_{\{i, j\} \subseteq[4]}\left|V_{i} \cap V_{j}\right| \leq n+8+8=n+16$. Furthermore, if equality holds, then for every vertex $v$ in $\Delta$, either $v \in V_{1} \cap V_{2}$, or $v \in V_{3} \cap V_{4}$, or $v \in \bar{V}$. This implies that every vertex in $\Delta$ belongs to at least two of the four links $\mathrm{lk}_{\Delta} v_{1}, \cdots, \mathrm{lk}_{\Delta} v_{4}$. This proves the second claim.

Lemma 5.3 Let $\Delta$ be a flag weak 3-pseudomanifold on $n$ vertices, and let $\sigma=\left\{v_{1}, v_{2}, v_{3}, v_{4}\right\}$ be an arbitrary facet of $\Delta$. Then $\sum_{1 \leq i \leq 4} f_{0}\left(\mathrm{lk}_{\Delta} v_{i}\right) \leq 2 n+8$. If equality holds, then $\cup_{w \in \tau} V\left(\mathrm{lk}_{\Delta} w\right)=V(\Delta)$ for any ridge $\tau \subseteq \sigma$.

Proof: The proof uses the inclusion-exclusion principle and Lemma 5.2 .

Now we are ready to prove the main result of this section.

Proof of Theorem 1.5: We denote the vertices of $\Delta$ by $v_{1}, v_{2}, \cdots, v_{n}$ and we let $a_{i}=f_{0}\left(\operatorname{lk}_{\Delta} v_{i}\right)$. Since $\mathrm{lk}_{\Delta} v_{i}$ is an Eulerian complex of dimension 2, the $f$-numbers of $\mathrm{lk}_{\Delta} v_{i}$ satisfy the relations

$$
f_{2}-f_{1}+f_{0}=2,3 f_{2}=2 f_{1} \text {. }
$$


Hence $f_{2}\left(\mathrm{lk}_{\Delta} v_{i}\right)=2 a_{i}-4$. By double counting, we obtain that

$$
\sum_{\sigma \in \Delta,|\sigma|=4} \sum_{v \in \sigma} f_{0}\left(\mathrm{lk}_{\Delta} v\right)=\sum_{i=1}^{n} f_{0}\left(\mathrm{lk}_{\Delta} v_{i}\right) \cdot \#\left\{\sigma: v_{i} \in \sigma,|\sigma|=4\right\}=\sum_{i=1}^{n} a_{i}\left(2 a_{i}-4\right) .
$$

By Lemma 5.3 the left-hand side of $(3)$ is bounded above by $f_{3}(\Delta)(2 n+8)$, which also equals $\left(f_{1}(\Delta)-\right.$ $n)(2 n+8)$ by Lemma 2.2 However, since $2 f_{1}(\Delta)=\sum_{i=1}^{n} f_{0}\left(\mathrm{lk}_{\Delta} v_{1}\right)$, the right-hand side of 3 is bounded below by $n \cdot \frac{2 f_{1}(\Delta)}{n} \cdot\left(\frac{4 f_{1}(\Delta)}{n}-4\right)$, and equality holds only if $a_{i}=\frac{2 f_{1}(\Delta)}{n}$ for all $1 \leq i \leq n$. Hence,

$$
\left(f_{1}(\Delta)-n\right)(2 n+8) \geq n \cdot \frac{2 f_{1}(\Delta)}{n} \cdot\left(\frac{4 f_{1}(\Delta)}{n}-4\right) .
$$

We simplify this inequality to get

$$
\left(f_{1}(\Delta)-n\right)\left(\frac{8}{n} f_{1}(\Delta)-(2 n+8)\right) \leq 0 .
$$

Since $f_{1}(\Delta) \geq n$, it follows that $f_{1}(\Delta) \leq\left\lfloor\frac{n^{2}}{4}\right\rfloor+n$, that is, $f_{1}(\Delta) \leq f_{1}\left(J_{2}(n)\right)$. Furthermore, if $f_{1}(\Delta)=\left\lfloor\frac{n^{2}}{4}\right\rfloor+n$, then there must be $\left\lceil\frac{n}{2}\right\rceil$ vertices such that $f_{0}\left(\mathrm{lk}_{\Delta} v\right)=\left\lfloor\frac{n}{2}\right\rfloor+2$, while the rest of vertices have $f_{0}\left(\mathrm{lk}_{\Delta} v\right)=\left\lceil\frac{n}{2}\right\rceil+2$. This proves our claim.

The following corollary provides some further properties of the maximizers of the face numbers in the class of 3-dimensional flag Eulerian complexes. (We omit the proof.)

Corollary 5.4 Let $\Delta$ be a 3-dimensional flag Eulerian complex on $n$ vertices. If $f_{1}(\Delta)=f_{1}\left(J_{2}(n)\right)$, then $\Delta$ and all of its vertex links are connected.

The next lemma provides a sufficient condition for a flag complex to be the join of two of its links. The proof simply relies on Lemma 2.3 and properties of normal pseudomanifolds; we omit it here.

Lemma 5.5 Let $\Delta$ be a flag $(d-1)$-dimensional simplicial complex. If $\sigma=\tau_{1} \cup \tau_{2}$ is a facet of $\Delta$, where $\tau_{1}$ is an $i$-face of $\Delta$ and $\tau_{2}$ is a $(d-i-2)$-face of $\Delta$, then $V\left(\mathrm{lk}_{\Delta} \tau_{1}\right) \cup V\left(\mathrm{k}_{\Delta} \tau_{2}\right)=V(\Delta)$ implies that $\Delta \subseteq \mathrm{lk}_{\Delta} \tau_{1} * \mathrm{lk}_{\Delta} \tau_{2}$. Moreover, if $\Delta$ is a flag normal $(d-1)$-pseudomanifold, then $V\left(\mathrm{lk}_{\Delta} \tau_{1}\right) \cup$ $V\left(\mathrm{lk}_{\Delta} \tau_{2}\right)=V(\Delta)$ if and only if $\Delta=\mathrm{lk}_{\Delta} \tau_{1} * \mathrm{lk}_{\Delta} \tau_{2}$.

Remark 5.6 The second result in Lemma 5.5 does not hold for flag weak pseudomanifolds, even assuming connectedness. Indeed, let $L_{1}, \cdots, L_{4}$ be four distinct circles of length $\geq 4$. Then $\Delta=\left(L_{1} * L_{3}\right) \cup\left(L_{2} *\right.$ $\left.L_{3}\right) \cup\left(L_{1} * L_{4}\right)$ is a flag weak 3-pseudomanifold. If $\tau_{1}$ and $\tau_{2}$ are edges in $L_{1}$ and $L_{3}$ respectively, then $\mathrm{lk}_{\Delta} \tau_{1}=L_{3} \sqcup L_{4}$ and $\mathrm{lk}_{\Delta} \tau_{2}=L_{1} \sqcup L_{2}$. Hence $V\left(\mathrm{lk}_{\Delta} \tau_{1}\right) \cup V\left(\mathrm{lk}_{\Delta} \tau_{2}\right)=V(\Delta)$. However, $\Delta$ is $a$ proper subcomplex of $\mathrm{lk}_{\Delta} \tau_{1} * \mathrm{lk}_{\Delta} \tau_{2}$.

In Theorem 1.3, we proved that the maximizer of the face numbers is unique in the class of flag 3manifolds on $n$ vertices. Is this also true for 3-dimensional flag Eulerian complexes? Corollary 5.4 implies that if the case of equality is not a join of two circles, then some of its edge links are not connected. Motivated by the example in Remark 5.6 we construct a family of 3-dimensional flag Eulerian complexes on $n$ vertices that have the same $f$-numbers as those of $J_{2}(n)$. 
Example 5.7 We write $C_{n}$ to denote a circle of length $n$. Let $a_{1}, a_{2}, \cdots, a_{s}, b_{1}, b_{2}, \cdots, b_{t} \geq 4$ be integers such that

$$
\sum_{1 \leq i \leq s} a_{i}=\left\lfloor\frac{n}{2}\right\rfloor, \text { and } \sum_{1 \leq j \leq t} b_{j}=\left\lceil\frac{n}{2}\right\rceil .
$$

We claim that $\Delta=\cup_{1 \leq i \leq s, 1 \leq j \leq t}\left(C_{a_{i}} * C_{b_{j}}\right)$ is flag and Eulerian, where all $C$. are defined on disjoint vertex sets. Since the circles $C_{a_{i}}$ and $C_{b_{j}}$ are of length $\geq 4$, it follows that $\Delta$ is flag. Also any ridge $\tau$ in $\Delta$ can be expressed as $\tau=\{v\} \cup e$, where $v \in C_{a_{i}}$ and the edge e $\in C_{b_{j}}$ (or $v \in C_{b_{j}}$ and $e \in C_{a_{i}}$ ) for some $i, j$. By the construction of $\Delta$, the ridge $\tau$ is contained in exactly two facets $\left\{v, v^{\prime}\right\} \cup e$ and $\left\{v, v^{\prime \prime}\right\} \cup e$ of $\Delta$, where $v^{\prime}$ and $v^{\prime \prime}$ are neighbors of $v$ in the circle $C_{a_{i}}\left(\right.$ or $\left.C_{b_{j}}\right)$. Hence the links of ridges in $\Delta$ are Eulerian. Since the edge links in $\Delta$ are either a circle or disjoint union of circles, and the vertex links in $\Delta$ are suspensions of disjoint union of circles, these links are also Eulerian. Finally, the vertices in $C_{a_{i}}$ have degree $\left\lceil\frac{n}{2}\right\rceil+2$ and the vertices in $C_{b_{j}}$ have degree $\left\lfloor\frac{n}{2}\right\rfloor$, and thus $f_{1}(\Delta)=f_{1}\left(J_{2}(n)\right)$. A simple computation also shows that $f_{2}(\Delta)=f_{2}\left(J_{2}(n)\right)$ and $f_{3}(\Delta)=f_{3}\left(J_{2}(n)\right)$. Hence $\chi(\Delta)=\chi\left(J_{2}(n)\right)=0$ and $\Delta$ is Eulerian.

We denote the set of all complexes on $n$ vertices constructed in Example 5.8 as $G J(n)$. It turns out that $G J(n)$ is exactly the set of maximizers of the face numbers in the class of flag 3-dimensional Eulerian complex on $n$ vertices. To prove this, we begin with the following lemma.

Lemma 5.8 Let $\Delta$ be a flag 3-dimensional Eulerian complex on $n$ vertices. If $f_{1}(\Delta)=f_{1}\left(J_{2}(n)\right)$, then every vertex link is the suspension of disjoint union of circles.

Proof: Applying the inclusion-exclusion principle on $V\left(\operatorname{lk}_{\Delta} v_{i}\right), 1 \leq i \leq 3$, where $v_{1}, v_{2}, v_{3}$ are the vertices of a 2 -face of $\Delta$, and then using properties of Eulerian complexes yields the result.

Theorem 5.9 Let $\Delta$ be a flag 3-dimensional Eulerian complex on $n$ vertices. If $f_{1}(\Delta)=f_{1}\left(J_{2}(n)\right)$, then $\Delta \in G J(n)$.

Proof: By Lemma 5.8, we may assume that the link of vertex $v_{1} \in \Delta$ is the join of $C$ and two other vertices $v_{2}, v_{3}$, where $C$ is the disjoint union of circles. Then again by Lemma 5.8, the link of vertex $v_{2}$ is also the suspension of $C$. If $v_{1}^{\prime}$ is any vertex of $C$ and its adjacent vertices in $C$ are $v_{2}^{\prime}, v_{3}^{\prime}$, then by Lemma 2.3. $\Delta[V(C)]=C$, and it follows that $f_{0}\left(\mathrm{lk}_{\Delta} v_{i}^{\prime} \cap C\right)=2$ for $i=1,2$. Hence for $1 \leq i, j \leq 2$,

$$
f_{0}\left(\mathrm{lk}_{\Delta}\left\{v_{i}, v_{j}^{\prime}\right\}\right)=f_{0}\left(\mathrm{lk}_{\Delta} v_{i} \cap \mathrm{lk}_{\Delta} v_{j}^{\prime}\right) \leq f_{0}\left(C \cap \mathrm{lk}_{\Delta} v_{j}^{\prime}\right)+2=4 .
$$

Furthermore, $V\left(\operatorname{lk}_{\Delta}\left\{v_{1}^{\prime}, v_{2}^{\prime}\right\}\right)$ is disjoint from $V\left(\operatorname{lk}_{\Delta}\left\{v_{1}, v_{2}\right\}\right)$. So we obtain that

$$
\sum_{e \subseteq\left\{v_{1}^{\prime}, v_{2}^{\prime}, v_{1}, v_{2}\right\}} f_{0}\left(\mathrm{lk}_{\Delta} e\right) \leq n+4 \cdot 4=n+16,
$$

where the sum is over the edges of $\left\{v_{1}^{\prime}, v_{2}^{\prime}, v_{1}, v_{2}\right\}$. Since $f_{1}(\Delta)=f_{1}\left(J_{2}(n)\right)$, by the proof of Theorem 1.5 and Lemma 5.2 it follows that this sum is exactly $n+16$. Hence $V\left(\operatorname{lk}_{\Delta}\left\{v_{1}, v_{2}\right\}\right) \cup V\left(\operatorname{lk}_{\Delta}\left\{v_{1}^{\prime}, v_{2}^{\prime}\right\}\right)=$ $V(\Delta)$. By Lemma 5.5, $\Delta \subseteq \operatorname{lk}_{\Delta}\left\{v_{1}, v_{2}\right\} * \operatorname{lk}_{\Delta}\left\{v_{1}^{\prime}, v_{2}^{\prime}\right\}$. We count the number of edges in $\Delta$ to get

$$
\begin{aligned}
f_{1}\left(J_{2}(n)\right)=f_{1}(\Delta) & \leq f_{1}\left(\mathrm{lk}_{\Delta}\left\{v_{1}, v_{2}\right\} * \mathrm{lk}_{\Delta}\left\{v_{1}^{\prime}, v_{2}^{\prime}\right\}\right) \\
& =f_{0}\left(\mathrm{lk}_{\Delta}\left\{v_{1}, v_{2}\right\}\right) \cdot f_{0}\left(\mathrm{lk}_{\Delta}\left\{v_{1}^{\prime}, v_{2}^{\prime}\right\}\right)+n \\
& \leq f_{1}\left(J_{2}(n)\right) .
\end{aligned}
$$


Thus $f_{1}(\Delta)=f_{1}\left(\mathrm{lk}_{\Delta}\left\{v_{1}, v_{2}\right\} * \mathrm{lk}_{\Delta}\left\{v_{1}^{\prime}, v_{2}^{\prime}\right\}\right)$, and the edge links $\operatorname{lk}_{\Delta}\left\{v_{1}, v_{2}\right\}$ and $\operatorname{lk}_{\Delta}\left\{v_{1}^{\prime}, v_{2}^{\prime}\right\}$ must be disjoint unions of circles on $\left\lceil\frac{n}{2}\right\rceil$ and $\left\lfloor\frac{n}{2}\right\rfloor$ vertices respectively. Since the flag complex $\Delta$ is determined by its graph, it follows that $\Delta=\operatorname{lk}_{\Delta}\left\{v_{1}, v_{2}\right\} * \mathrm{lk}_{\Delta}\left\{v_{1}^{\prime}, v_{2}^{\prime}\right\}$, i.e., $\Delta \in G J(n)$.

Remark 5.10 Theorem 5.9 implies Theorem 1.3 This is because every 3-manifold is Eulerian and the only complex in $G J(n)$ that is also a 3-manifold is $J_{2}(n)$.

Remark 5.11 The complexes from Example 5.8 form asymptotically the complete list of maximizers of the number of edges in the class of $K_{1,3,3}$-free graphs, see (Simonovits. 1966. Theorem 5). (Here $K_{r_{1}, r_{2}, \cdots, r_{m}}$ denotes the complete m-partite graph with $r_{i}$ vertices of color $i$. ) A more general result on extremal graphs not containing $K_{r_{1}, r_{2}, \cdots, r_{m}}$ can be found in Erdös and Simonovits (1972). Studying these extremal graphs is the main tool of Adamaszek and Hladky's work Adamaszek and Hladky (2015) on asymptotic upper bounds.

\section{Concluding Remarks}

We close this paper with a few remarks and open problems.

As mentioned in the introduction, Klee (1964) verified that the Motzkin's UBC for polytopes holds for Eulerian complexes with sufficiently many vertices, and conjectured it holds for all Eulerian complexes. Can the flag upper bounds for spheres also be extended to Eulerian complexes? Motivated by Theorem 1.3 and Theorem 1.5, we posit the following conjecture in the same spirit as Problem 17(i) from Adamaszek and Hladký (2015):

Conjecture 6.1 Let $\Delta$ be a flag $(2 m-1)$-dimensional complex, where $m \geq 2$. Assume further that $\Delta$ is an Eulerian complex on $n$ vertices. Then $f_{i}(\Delta) \leq f_{i}\left(J_{m}(n)\right)$ for all $i=1, \cdots, 2 m-1$.

Theorem 1.5 gives an affirmative answer in the case of $m=2$ and $1 \leq i \leq 3$. The next case is $i=1$ and $m=3$. In this case, Theorem 1.4 verifies Conjecture 6.1 for flag 5-manifolds. At present other cases are completely open.

The above results and conjectures discuss odd-dimensional flag complexes. What happens in the evendimensional cases? To this end, we pose the following strengthening of Conjecture 18 from Adamaszek and Hladký (2015).

Recall that $J_{m}^{*}(n)=\mathbb{S}^{0} * C_{1} * \cdots * C_{m}$, where $n \geq 4 m+2$, each $C_{i}$ is a circle of length either $\left\lceil\frac{n-2}{m}\right\rceil$ or $\left\lfloor\frac{n-2}{m}\right\rfloor$ so that the total number of vertices of $J_{m}^{*}(n)$ is $n$. Now we let $\mathcal{S}_{n}$ denote the set of flag 2-spheres on $n$ vertices, and define

$$
\mathcal{J}_{m}^{*}(n):=\left\{S * C_{2} * \cdots * C_{m} \mid S \in \mathcal{S}_{V\left(C_{1}\right)+2}\right\} .
$$

It is not hard to see that every element in $\mathcal{J}_{m}^{*}(n)$ is a flag $2 m$-sphere.

Conjecture 6.2 Let $\Delta$ be a flag homology $2 m$-sphere on $n$ vertices. Then $f_{i}(\Delta) \leq f_{i}\left(J_{m}^{*}(n)\right)$ for all $i=1, \cdots, 2 m$. If equality holds for some $1 \leq i \leq 2 m$, then $\Delta \in \mathcal{J}_{m}^{*}(n)$.

\section{Acknowledgements}

I would like to thank Michal Adamaszek, Eran Nevo, Isabella Novik, Steven Klee and the referees for helpful comments. 


\section{References}

M. Adamaszek. An upper bound theorem for a class of flag weak pseudomanifolds. 2013. URL http: //arxiv.org/pdf/1303.5603v1.pdf.

M. Adamaszek and J. Hladký. Upper bound theorem for odd-dimensional flag manifolds. 2015. URL http://arxiv.org/pdf/1503.05961v2.pdf.

B. Bagchi and B. Datta. Minimal triangulations of sphere bundles over the circle. J. Combin. Theory Ser. A, 115:737-752, 2008. ISSN 0097-3165.

R. Charney and M. Davis. The Euler characteristic of a nonpositively curved, piecewise Euclidean manifold. Pacific J. Math., 171:117-137, 1995.

M. Davis and B. Okun. Vanishing theorems and conjectures for the $L^{2}$-homology of right-angled Coxeter groups. Geom. Topol., 5:7-74, 2001. ISSN 1465-3060.

M. Davis, J. Dymara, T. Januszkiewicz, and B. Okun. Weighted $L^{2}$-cohomology of Coexter groups. Geom. Topol., 11:47-138, 2007. ISSN 1465-3060.

P. Erdös and M. Simonovits. An extremal graph problem. Acta Mathematica Academiae Scientiarum Hungarica, 22:275-282, 1972.

P. Frankl, Z. Füredi, and G. Kalai. Shadows of colored complexes. Math. Scand., 63:169-178, 1988. ISSN 1903-1807.

S. Gal. Real root conjecture fails for five and higher dimensional spheres. Discrete Comput. Geom., 34 : 269-284, 2005. ISSN 0179-5376.

V. Klee. On the number of vertices of a convex polytope. Canadian J. Math, 16:702-720, 1964.

F. Lutz and E. Nevo. Stellar theory for flag complexes. Math. Scand. to appear, 2014. URL http: //arxiv.org/pdf/1302.5197v3.pdf.

P. McMullen. The maximum number of faces of a convex polytope. Mathematika, 17:179-184, 1970. ISSN 2041-7942.

T. Motzkin. Comonotone curves and polyhedra. Bull. Amer. Math. Soc., 63:35, 1957.

E. Nevo and T. K. Petersen. On $\gamma$-vectors satisfying the Kruskal-Katona inequalities. Discrete Comput. Geom., 45:503-521, 2010. ISSN 0179-5376.

A. Padrol. Many neighborly polytopes and oriented matroids. Discrete Comput. Geom., 50:865-902, 2013. ISSN 0179-5376.

I. Shermer. Neighborly polytopes. Israel J. Math., 43:291-314, 1982. ISSN 0021-2172.

M. Simonovits. A method for solving extremal problems in graph theory, stability problems. Theory of graphs, Pro. Coll. held at Tihany, Hungary, 1966.

R. Stanley. The upper bound conjecture and Cohen-Macaulay rings. Studies in Applied Math., 54:135142, 1975. ISSN 1467-9590. 
